\title{
Previsões meteorológicas do Modelo Eta para subsidiar o uso de modelos de previsão agrícola no Centro-Sul do Brasil
}

\author{
Eta model forecasts as input to crop models for the Mid-Southern region of Brazil
}

\author{
Pedro Abel Vieira JuniorI Durval Dourado Neto ${ }^{\mathrm{II}}$ Sin Chan Chou ${ }^{\mathrm{III}}$ Thomas Newton Martin $^{\mathrm{IV}}$
}

RESUMO

Este trabalho tem por objetivo avaliar a precipitação pluvial e as temperaturas máximas e mínimas previstas pelo Modelo Eta para até 120 dias de previsão em 24 localidades distribuídas na região Centro-Sul do Brasil. A avaliação se baseia na comparação de séries históricas de chuva e temperaturas de 1997 a 2002, com as previsões de 30 , 60 e 120 dias de antecedência do Modelo Eta para as 24 localidades. Foram utilizados valores de média, mediana e desvio padrão nesta avaliação. Os resultados indicam que estas previsões geralmente subestimam as chuvas e a amplitude térmica nestas localidades. Os menores erros de precipitação pluvial se localizam mais destacadamente em Itumbiara e Rio Verde, enquanto que os maiores, em Porangatu e Manduri. Por outro lado, os menores erros de temperatura máxima se localizam destacadamente em Brasília, Manduri e Piracicaba, enquanto que os maiores ocorrem em Barreiras e Porangatu. Apesar de o modelo apresentar erros sistemáticos nas previsões de temperaturas, estes erros podem ser removidos para que os valores corrigidos possam ser introduzidos nos modelos de culturas.

Palavras-chave: previsões meteorológicas, aplicação modelo de cultura.

\section{ABSTRACT}

The objective of this study was to evaluate the 120 day precipitation and maximum and minimum temperature forecasts by Eta Model over 24 locations around the MidSouthern of Brazil. The evaluation was based on comparing observed time series of precipitation and temperatures from 1997 to 2002 to 30, 60 and 120-day forecasts of Eta Model over these 24 locations. Mean, median and standard deviation were used in the evaluation. The results show that these forecasts generally underestimate rain and temperature range. The smallest precipitation errors occurred in Itumbiara and Rio Verde, whereas the largest errors occurred in Porangatu and Manduri. The smallest maximum temperature errors occurred in Brasília, Manduri and Piracicaba whereas the largest errors in Barreiras and Porangatu. Despite the systematic errors exhibited by the precipitation and temperature forecasts, these errors can be removed and the corrected values input into the crop models.

Key words: climate forecasts, applications to crop models.

\section{INTRODUÇÃO}

O Modelo Eta foi desenvolvido pela Universidade de Belgrado e o Instituto Hidrometeorológico da antiga Iugoslávia e posteriormente foi operacionalizado pelo National Center for Environmental Prediction (NCEP). No Brasil, o Modelo Eta foi instalado no Centro de Previsão de Tempo e Estudos Climáticos (CEPTEC) em 1996 (CHOU, 1996). Originalmente as previsões do Eta se estendiam por 48 horas, sendo geradas duas vezes ao dia, às $0 \mathrm{~h} e$ às $12 \mathrm{~h}$ UTC. Na América do Sul, o Modelo Eta tem sido utilizado para estudos de diferentes fenômenos meteorológicos como, por exemplo: jatos de baixos níveis (SAULO et al., 2000), trocas de massas de ar

IEmbrapa, Serviço de Negócios para Transferência de Tecnologia. E-mail: pavieira@cnpms.embrapa.br.

IIDepartamento de Produção Vegetal, Escola Superior de Agricultura (ESALQ), Universidade de São Paulo (USP), Piracicaba, SP, Brasil. E-mail: dourado@esalq.usp.br

IIICentro de Previsão de Tempo e Estudos Climáticos do Instituto Nacional de Pesquisas Espaciais. E-mail: chou@cptec.inpe.br.

${ }^{\text {IV} U n i v e r s i d a d e ~ T e c n o l o ́ g i c a ~ F e d e r a l ~ d o ~ P a r a n a ́, ~ C a m p u s ~ D o i s ~ V i z i n h o s . ~ T u t o r ~ G r u p o ~ P E T-Z o o t e c n i a, ~ C P ~ 157, ~ 85660-000, ~ D o i s ~}$

Vizinhos, PR, Brasil. Autor para correspondência 
(SELUCHI \& CHOU, 2003) e vento Zonda (SELUCHI et al., 2003). Para previsões de precipitação até três dias de antecedência, o modelo tem apresentado índices de acerto superior aos das previsões do modelo global do CPTEC (CHOU \& SILVA, 1999; CHOU et al., 2004). Para previsões meteorológicas sazonais, isto é, de até 120 dias de antecedência, as avaliações apresentam índices de acerto menor que as previsões para até três dias de antecedência porque os erros de previsão crescem com o prazo da integração do modelo. Entretanto, as previsões de até 120 dias de antecedência ainda se apresentam úteis, principalmente, quando usadas na forma de médias para estação (CHOU et al., 2005). Como exemplo de aplicação, encontra-se as previsões em pontos localizados em sete cidades da região Nordeste (CHOU et al., 2007).

O Modelo Eta é um modelo estado da arte para pesquisa ou uso operacional em meteorologia. O modelo representa o estado atmosférico em pontos de grade, sendo configurado na resolução de $40 \mathrm{~km}$ na horizontal e 38 níveis na vertical. Uma das principais vantagens do modelo é a coordenada vertical e a estabilidade numérica (MESINGER et al., 1990). As variáveis prognósticas do modelo são: temperatura do ar, umidade, pressão à superfície, vento horizontal, energia cinética turbulenta e água líquida ou gelo das nuvens previstas em intervalos de seis horas. A condição inicial para o Eta é fornecida pelo NCEP e as condições de contorno lateral, atualizadas a cada 6 horas, são as previsões do modelo global do CPTEC.

Aplicação das previsões meteorológicas sazonais no setor energético para planejamento de operação do sistema elétrico nacional tem sido bastante explorada e as previsões avaliadas. No setor agrícola, estas previsões ainda são pouco aproveitadas no seu valor numérico, pois há a possibilidade de utilizá-las associadas a modelos de cultura para estimativa da produção de várias espécies agrícolas de interesse, a exemplo do milho.

É importante a estimativa dos erros das previsões de variáveis climáticas nesta fase antes da aplicação em modelos de cultura, visto que as interações destas previsões com modelos de cultura podem ser não-lineares e os erros dos resultados se tornam de difícil identificação. Considerando o exposto, este trabalho tem por objetivo avaliar a precipitação pluvial e as temperaturas máximas e mínimas previstas pelo Modelo Eta para até 120 dias de previsão em 24 localidades distribuídas na região Centro-Sul do Brasil visando à aplicação em estimativas de modelos de rendimento agrícola.

\section{MATERIAL E MÉTODOS}

O Modelo Eta foi iniciado no dia 15 de cada mês e integrado para produzir previsões de 120 dias. Foram geradas previsões em intervalos de seis horas entre junho de 1997 e abril de 2002, totalizando 49 previsões de 120 dias cada neste período. Os elementos meteorológicos de interesse foram: i) precipitação pluvial (mm), densidade de fluxo de radiação solar global incidente $\left(\mathrm{W} \mathrm{m}^{-2}\right)^{\mathrm{b}}$ e iii) temperatura do ar $\left({ }^{\circ} \mathrm{C}\right)$; foram fornecidas em grade regular de $40 \mathrm{~km}$ e estimativas foram realizadas com 30, 60, 90 e 120 dias de antecedência. A primeira previsão foi realizada às $12 \mathrm{~h}$ UTC do dia 16 de julho de 1997, sendo que foram realizadas quatro previsões contadas a partir das $12 \mathrm{~h}$ UTC dos dias 16 de junho de 1997, 17 de maio de 1997, 17 de abril de 1997 e 18 de março de 1997, respectivamente. Para verificação da acurácia das previsões geradas pelo Modelo Eta, foram realizadas comparações entre os valores medidos de precipitação pluvial, as temperaturas máxima e mínima e os respectivos valores previstos na base diária. Para tanto, foram utilizados dados meteorológicos provindos de estações meteorológicas convencionais e automáticas dispostas nos locais avaliados, conforme indicado na tabela 1. A comparação ideal entre os parâmetros atmosféricos estimados e as medidas requer a obtenção de valores de avaliação dos parâmetros atmosféricos, o que permitiria estimar a probabilidade de acerto das previsões geradas pelo Modelo Eta. Para tanto, foram calculadas as médias e as medianas entre o dia 16 de Julho de 1997 e 15 de Junho de 2002 da precipitação pluvial e das temperaturas máximas e mínimas diárias medidas e previstas pelo Modelo Eta. A seguir, foram calculadas as variações em relação à média para o conjunto de parâmetros medidos e previstos do período entre o dia 16 de Julho de 1997 e 15 de Junho de 2002 como análise exploratória das previsões geradas pelo Modelo Eta. As médias destas variações são denominadas como desvio médio dos parâmetros.

Também foi calculada a razão entre os valores previstos pelo Eta e os respectivos valores medidos no período de previsões de 16 de Julho de 1997 a 15 de Junho de 2002, denominada erro sistemático relativo ou viés relativo, entre os valores previstos e os medidos. Para tanto, foram calculados os erros sistemáticos relativos entre os valores diários dos parâmetros atmosféricos previstos pelo Modelo Eta com 30, 60, 90 e 120 dias de antecedência em relação aos respectivos valores medidos nos locais indicados na tabela 1, determinando-se assim a acurácia dessas previsões meteorológicas com até 120 dias de antecedência, em intervalos regulares de 30 dias. A 
Tabela 1 - Coordenadas geográficas das localidades com disponibilidade de ensaios de rendimento de grãos de milho. A disponibilidade dos dados está indicada por (O).

\begin{tabular}{|c|c|c|c|c|c|c|c|c|c|c|}
\hline UF & Local & Instituição & Lat. & Long. & Altitude & & & SAFRAS & & \\
\hline & & & $\left({ }^{\circ} \mathrm{S}\right)$ & $\left({ }^{\circ} \mathrm{W}\right)$ & (m) & 97_98 & 98_99 & 99_00 & 00_01 & 01_02 \\
\hline $\mathrm{BA}$ & Barreiras* & INMET & 12,09 & 45,01 & 800 & & $\mathrm{O}$ & $\mathrm{O}$ & $\mathrm{O}$ & \\
\hline DF & Brasília & INMET & 15,47 & 47,56 & 1.000 & & $\mathrm{O}$ & $\mathrm{O}$ & $\mathrm{O}$ & $\mathrm{O}$ \\
\hline \multirow[t]{5}{*}{ GO } & Goianésia & INMET & 15,13 & 49 & 640 & & & $\mathrm{O}$ & & $\mathrm{O}$ \\
\hline & Goiânia & CNPAF & 16,4 & 49,15 & 749 & & $\mathrm{O}$ & $\mathrm{O}$ & $\mathrm{O}$ & $\mathrm{O}$ \\
\hline & Itumbiara & INMET & 18,25 & 49,13 & 420 & $\mathrm{O}$ & $\mathrm{O}$ & $\mathrm{O}$ & $\mathrm{O}$ & \\
\hline & Porangatu & SIMEGO & 13,27 & 49,1 & 396 & & $\mathrm{O}$ & $\mathrm{O}$ & $\mathrm{O}$ & $\mathrm{O}$ \\
\hline & Rio Verde & INMET & 17,48 & 50,55 & 715 & & $\mathrm{O}$ & $\mathrm{O}$ & & \\
\hline MS & Campo Grande & INMET & 20,26 & 54,43 & 484 & & $\mathrm{O}$ & & & $\mathrm{O}$ \\
\hline \multirow[t]{8}{*}{ MG } & Capinópolis & INMET & 18,43 & 49,43 & 620 & $\mathrm{O}$ & $\mathrm{O}$ & $\mathrm{O}$ & $\mathrm{O}$ & \\
\hline & Janaúba & UFV & 15,47 & 43,18 & 530 & & & $\mathrm{O}$ & & \\
\hline & Lavras & INMET & 21,14 & 45 & 940 & $\mathrm{O}$ & $\mathrm{O}$ & $\mathrm{O}$ & $\mathrm{O}$ & \\
\hline & Paracatu & UFLA & 17,13 & 46,52 & 687 & $\mathrm{O}$ & & & $\mathrm{O}$ & \\
\hline & Patos de Minas & INMET & 18,31 & 46,26 & 900 & & $\mathrm{O}$ & $\mathrm{O}$ & $\mathrm{O}$ & $\mathrm{O}$ \\
\hline & Sete Lagoas & INMET & 19,28 & 44,15 & 740 & & $\mathrm{O}$ & $\mathrm{O}$ & $\mathrm{O}$ & $\mathrm{O}$ \\
\hline & Uberlândia & CNPMS & 18,55 & 48,15 & 830 & & $\mathrm{O}$ & $\mathrm{O}$ & $\mathrm{O}$ & $\mathrm{O}$ \\
\hline & Viçosa & INMET & 21,02 & 42,33 & 720 & & $\mathrm{O}$ & $\mathrm{O}$ & $\mathrm{O}$ & $\mathrm{O}$ \\
\hline \multirow[t]{5}{*}{ SP } & Cravinhos & IAC & 21,01 & 47,46 & 590 & $\mathrm{O}$ & $\mathrm{O}$ & $\mathrm{O}$ & & \\
\hline & Guaíra & IAC & 20,33 & 47,26 & 450 & & $\mathrm{O}$ & $\mathrm{O}$ & $\mathrm{O}$ & $\mathrm{O}$ \\
\hline & Limeira & IAC & 22,37 & 47,32 & 560 & & $\mathrm{O}$ & $\mathrm{O}$ & $\mathrm{O}$ & $\mathrm{O}$ \\
\hline & Manduri & IAC & 23,02 & 49,1 & 590 & & $\mathrm{O}$ & $\mathrm{O}$ & $\mathrm{O}$ & $\mathrm{O}$ \\
\hline & Piracicaba & ESALQ & 22,43 & 47,38 & 547 & $\mathrm{O}$ & $\mathrm{O}$ & $\mathrm{O}$ & $\mathrm{O}$ & \\
\hline \multirow[t]{3}{*}{ PR } & Cascavel & SIMEPAR & 24,56 & 53,26 & 760 & $\mathrm{O}$ & $\mathrm{O}$ & $\mathrm{O}$ & & $\mathrm{O}$ \\
\hline & Londrina & SIMEPAR & 23,19 & 51,08 & 630 & & $\mathrm{O}$ & & $\mathrm{O}$ & $\mathrm{O}$ \\
\hline & Palotina & SIMEPAR & 24,18 & 53,55 & 333 & & & & $\mathrm{O}$ & \\
\hline
\end{tabular}

* Dados fornecidos por: Centro Nacional de Pesquisa de Milho e Sorgo, Centro Nacional de Arroz e Feijão, (ambos da Empresa Brasileira de Pesquisa Agropecuária), Escola Superior de Agricultura "Luiz de Queiroz", Instituto Agronômico de Campinas, Instituto Nacional de Meteorologia, Sistema Meteorológico de Goiás, Instituto Tecnológico, Universidade Federal de Lavras e Universidade Federal de Viçosa.

mediana e a amplitude das variações foram calculadas. As médias e as medianas foram calculadas para a série de cada localidade. Como o Modelo Eta gera parâmetros atmosféricos em intervalos de seis horas, foram somadas as quatro estimativas de precipitações pluviais para a obtenção da precipitação pluvial diária (é realizado o somatório dos valores estimados às 6,12 , 18 e 24 horas). Para as temperaturas do ar, foram consideradas as maiores e as menores estimativas previstas diárias como as temperaturas do ar máxima e mínima, respectivamente.

\section{RESULTADOS E DISCUSSÃO}

Para as previsões meteorológicas sazonais de precipitação pluvial, no que diz respeito ao desvio médio realizados pelo Modelo Eta (entre os dias 16 de julho de 1997 e 15 de junho de 2002), houve uma tendência de minimizar as variações entre os dias quando comparadas às variações medidas. Quando considerados todos os locais (Figura 1, Figura 2 e Figura
3), o desvio médio das medidas de precipitação pluvial $(10,4 \mathrm{~mm})$ na série em questão (temperaturas do ar) foi superior aos desvios médios das previsões de precipitação pluvial com 30 (7,6mm), 60 (6,7mm), 90 $(6,4 \mathrm{~mm})$ e $120(6,0 \mathrm{~mm})$ dias de antecedência. Estas previsões apresentaram redução das amplitudes proporcionalmente ao período de antecedência da previsão. No caso das previsões de temperatura do ar pelo Modelo Eta, embora não tão acentuada como na precipitação pluvial, observou-se uma ligeira redução das amplitudes térmicas com o aumento da antecedência da previsão, sendo que isso ocorreu em todas as localidades (Figura 1, Figura 2 e Figura 3). O desvio médio das medidas de temperaturas máximas do $\operatorname{ar}\left(3,5^{\circ} \mathrm{C}\right)$ foi ligeiramente superior ao desvio médio das previsões com 30 dias de antecedência $\left(3,4^{\circ} \mathrm{C}\right)$ e igual ao desvio médio das previsões com 60, 90 e 120 dias de antecedência. Por outro lado, no caso das temperaturas mínimas do ar, ocorreu aumento dos desvios com a antecedência da previsão, visto que o desvio médio das medidas de temperaturas mínimas 

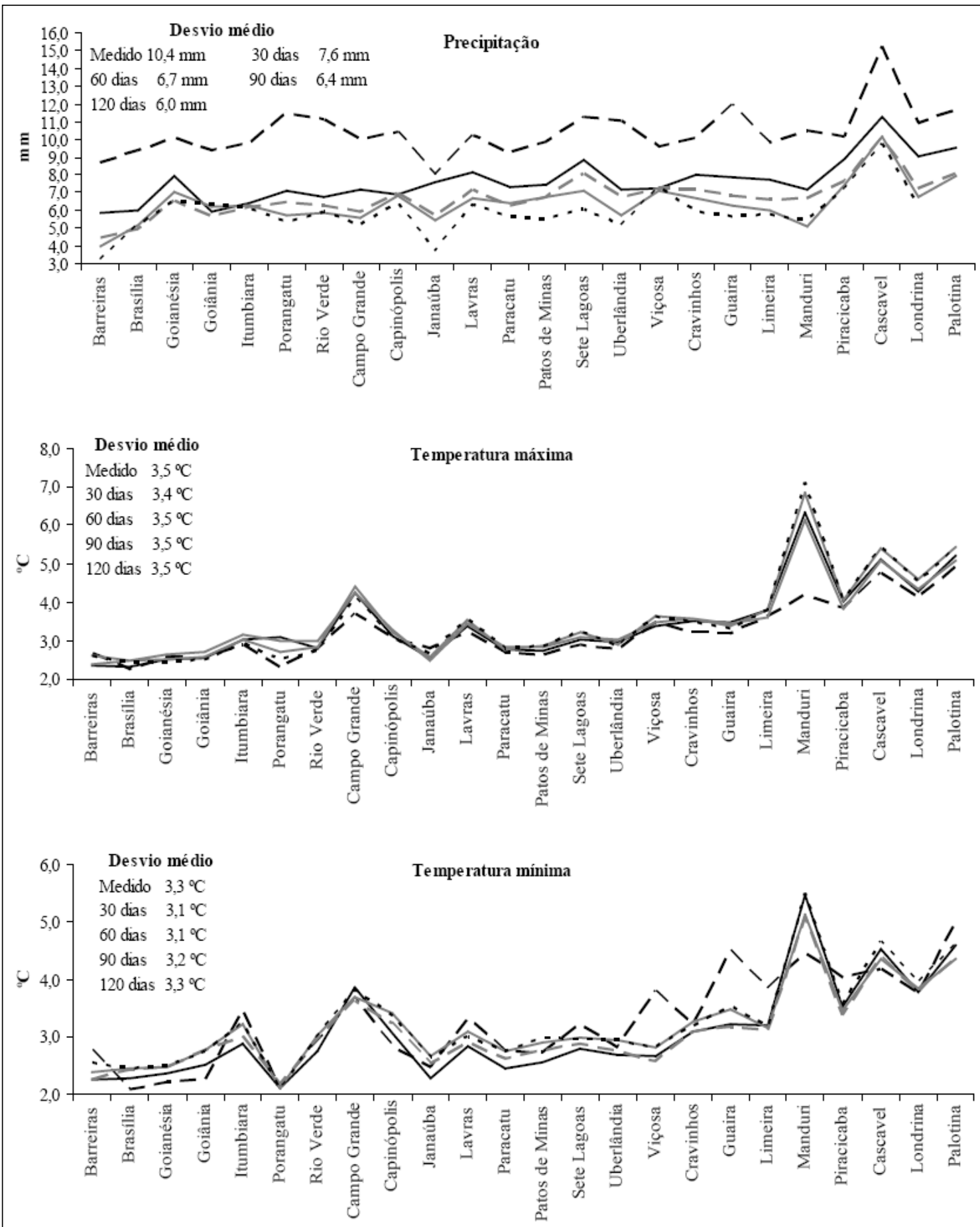

Figura 1 - Desvio médio entre medidas em 24 localidades (__ — $(\longrightarrow), 60$ ( - ), 90 ( ) e 120 ( -------- ) dias de antecedência da precipitação pluvial $(\mathrm{mm})$, temperatura máxima do ar $\left({ }^{\circ} \mathrm{C}\right)$ e temperatura mínima do ar $\left({ }^{\circ} \mathrm{C}\right)$.

do ar $\left(3,3^{\circ} \mathrm{C}\right)$ foi igual à previsão com 120 dias de antecedência e ligeiramente superior às previsões com $30\left(3,1^{\circ} \mathrm{C}\right), 60\left(3,1^{\circ} \mathrm{C}\right)$ e $90\left(3,2^{\circ} \mathrm{C}\right)$. Portanto, enquanto a variabilidade da série da precipitação medida é maior que a prevista, no caso das temperaturas do ar, a variabilidade das séries medidas é bastante próxima da variabilidade das séries previstas de temperaturas do ar.

Ciência Rural, v.39, n.2, mar-abr, 2009. 


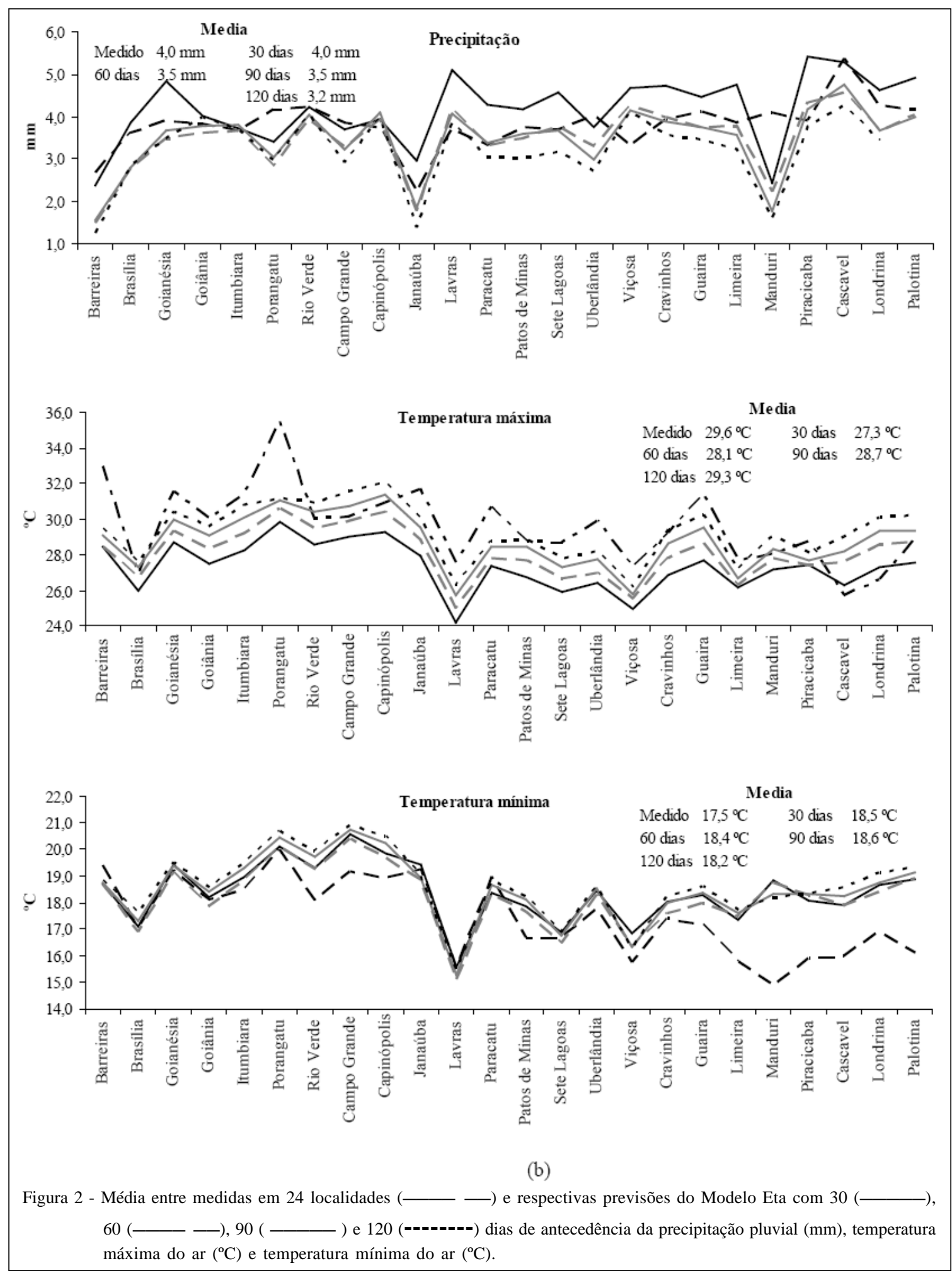

As previsões meteorológicas do Modelo Eta no período do estudo, quando observadas as precipitações pluviais médias diárias (Figura 1, Figura 2 e Figura 3) dos 24 locais, subestimaram as precipitações pluviais medidas. A média das precipitações pluviais medidas $(4,0 \mathrm{~mm})$ foi igual ou superior às respectivas médias estimadas com 30 (4,0mm), 60 (3,5mm), 90 (3,5mm) e 120 (3,2mm) dias de

Ciência Rural, v.39, n.2, mar-abr, 2009. 

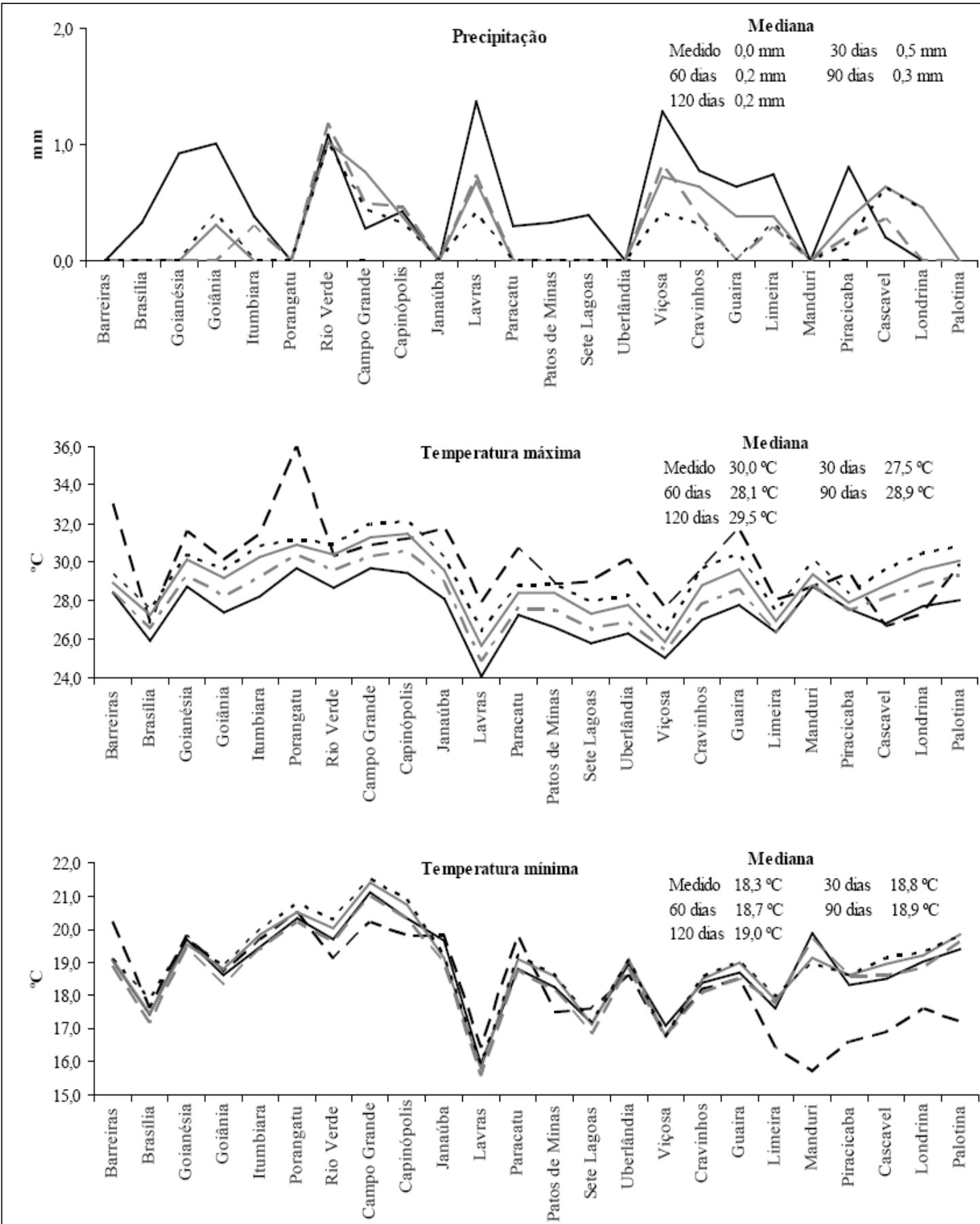

(c)

Figura 3 - Mediana entre medidas em 24 localidades

60 ( - -), 90 (— máxima do ar $\left({ }^{\circ} \mathrm{C}\right)$ e temperatura mínima do ar $\left({ }^{\circ} \mathrm{C}\right)$.

antecedência. A subestimação da previsão climática da precipitação pluvial pelo Modelo Eta não é corroborada pelas medianas (Figura 1, Figura 2 e Figura
3) das precipitações pluviais medidas $(0,0 \mathrm{~mm})$, que foi inferior às medianas estimadas com $30(0,5 \mathrm{~mm}), 60$ (0,2mm), 90 (0,3mm) e 120 (0,2mm) dias de antecedência,

Ciência Rural, v.39, n.2, mar-abr, 2009. 
ou seja, a maior precipitação pluvial não é resultado exclusivo de valores extremos, e sim de erros na previsão em todo período considerado.

No caso das temperaturas do ar, houve subestimação das máximas e superestimativa das mínimas, corroborando a menor amplitude nos desvios verificada para as temperaturas do ar previstas em relação à amplitude das temperaturas do ar medidas. A média das temperaturas do ar máximas $\left(29,6^{\circ} \mathrm{C}\right)$ medidas foi superior às médias das temperaturas máximas do ar previstas com $30\left(27,3^{\circ} \mathrm{C}\right), 60\left(28,1^{\circ} \mathrm{C}\right), 90\left(28,7^{\circ} \mathrm{C}\right)$ e 120 $\left(29,3^{\circ} \mathrm{C}\right)$ dias de antecedência. Ao contrário das previsões de precipitação, as previsões de temperatura máxima do ar se aproximam das observações com aumento da antecedência da previsão. Por outro lado, a média das temperaturas do ar mínimas $\left(17,5^{\circ} \mathrm{C}\right)$ medidas foram inferiores às médias das temperaturas mínimas do ar previstas com $30\left(18,4^{\circ} \mathrm{C}\right), 60\left(18,2^{\circ} \mathrm{C}\right), 90\left(18,5^{\circ} \mathrm{C}\right)$ e $120\left(18,6^{\circ} \mathrm{C}\right)$ dias de antecedência (Figura 1, Figura 2 e Figura 3). De modo semelhante à precipitação pluvial, essas médias não são afetadas por valores extremos, pois a mediana das temperaturas máximas do $\operatorname{ar}\left(30,0^{\circ} \mathrm{C}\right)$ medidas também foi superior às respectivas medianas previstas com $30\left(27,5^{\circ} \mathrm{C}\right), 60\left(28,1^{\circ} \mathrm{C}\right), 90\left(28,9^{\circ} \mathrm{C}\right)$ e 120 $\left(29,5^{\circ} \mathrm{C}\right)$ dias de antecedência, enquanto que a mediana $\left(18,3^{\circ} \mathrm{C}\right)$ das temperaturas mínimas do ar medidas foram inferiores às medianas previstas com $30\left(18,8^{\circ} \mathrm{C}\right), 60$ $\left(18,7^{\circ} \mathrm{C}\right), 90\left(18,9^{\circ} \mathrm{C}\right)$ e $120\left(19,0^{\circ} \mathrm{C}\right)$ dias de antecedência (Figura 1, Figura 2 e Figura 3), indicando a menor amplitude térmica prevista pelo modelo.

Há que se considerar que os resultados obtidos corroboram a normalização das previsões diárias de precipitação pluvial e temperatura do ar, notadamente a subestimativa e a superestimativa das temperaturas máximas e mínimas do ar, respectivamente, comentadas por DERECZYNSKI et al. (2000) e CHOU et al. (2005). Assim, a partir dos resultados obtidos, é possível inferir que as previsões do Modelo Eta, por tratar da interação entre a circulação geral na atmosfera e a topografia (CHOU et al., 2000), apresentam deficiências na modelagem dos elementos da circulação da atmosfera, dos elementos da topografia, da cobertura da superfície e das possíveis interações entre estes elementos. A correta descrição da superfície e da altitude da localidade permite melhor representar as variações de temperatura do ar.

Consideradas as previsões geradas pelo Modelo Eta com ênfase na estimativa de rendimento agrícola, notadamente no caso de culturas anuais, é possível inferir que a normalização dos parâmetros meteorológicos previstos pelo Modelo Eta afeta os resultados dos modelos de cultura. Para a cultura do milho, na região Centro-Sul do Brasil, é possível deduzir o efeito positivo das previsões do Modelo Eta sobre a estimativa do rendimento de grãos, pois, apesar da menor precipitação pluvial média prevista, esta é bem distribuída no tempo, além da menor amplitude diurna das temperaturas extremas do ar. Segundo DUARTE \& PATERNIANI (2000), estas condições são favoráveis ao maior rendimento de grãos da cultura. Entretanto, estão sendo analisados os erros das previsões de precipitação pluvial e a temperatura do ar do Modelo Eta em período de tempo amplo, compreendendo todas as estações do ano.

Uma forma adequada de aplicação das séries de temperatura do ar prevista em modelos de rendimento é por meio da remoção do erro sistemático da série prevista. O erro é obtido da diferença: (valor médio previsto - valor médio observado). Quando o erro sistemático, $\varepsilon$, é positivo, indica que a previsão sistematicamente superestima as observações em quantidade $\varepsilon$. Este erro pode ser diferente em cada antecedência de previsão. Por exemplo, para a série prevista de temperatura máxima do ar (Figura 1, Figura 2 e Figura 3), em Sete Lagoas, MG, é preciso remover em 30 dias de previsão cerca de $3,0^{\circ} \mathrm{C}$, em 90 dias cerca de $1,5^{\circ} \mathrm{C}$ e em 120 dias cerca de $1,0^{\circ} \mathrm{C}$ da série prevista. De modo geral, as previsões geradas pelo Modelo Eta foram acuradas, pois, à exceção de Barreiras, Manduri e Porangatu, as previsões dos demais locais podem ser consideradas adequadas para a previsão do rendimento de grãos de milho por modelo de cultura. Porém, apesar de serem consideradas acuradas, as previsões são influenciadas por características locais, pois algumas localidades do Estado de São Paulo, apesar de estarem situadas próximas, como Manduri e Piracicaba, apresentaram comportamentos distintos. Enquanto que em Manduri a tendência foi de prever precipitações pluviais menores que as respectivas precipitações pluviais medidas, em Piracicaba a tendência foi de prever precipitações pluviais maiores que as medidas, o que implica limitações na extrapolação e/ou na inferência de resultados obtidos nos locais vizinhos à localidade. As temperaturas mínimas do ar em São Paulo foram geralmente superestimadas nas previsões, principalmente, as de Limeira, Manduri e Piracicaba.

As previsões de precipitação pluvial geradas pelo Modelo Eta para os locais considerados de São Paulo também tenderam a ser menores e melhor distribuídas temporalmente que as respectivas precipitações pluviais medidas, especialmente em Manduri. Já quanto à temperatura do ar, as previsões geradas pelo Modelo Eta tendem a aumentar as variações entre os dias, porém minimizando as variações durante os dias em razão dos maiores valores previstos 
para as temperaturas mínimas do ar. Os resultados das precipitações pluviais e das temperaturas do ar previstas indicam que, com exceção de Manduri, o uso das previsões desses parâmetros meteorológicos não deverão afetar significativamente a previsão do rendimento de grãos de milho por modelo de cultura em São Paulo. Aponta-se ainda no mesmo Estado o efeito local sobre as previsões marcantes, o que implica a reduzida possibilidade da extrapolação de resultados.

As localidades no Estado do Paraná apresentaram as maiores variações entre os dias de precipitação pluvial e de temperatura do ar medidos. No caso das previsões de precipitação pluvial realizadas pelo Modelo Eta no Estado do Paraná, observa-se redução da variabilidade entre os dias da precipitação pluvial e redução proporcional ao aumento do período de antecedência da previsão. Também foi verificada a tendência das previsões de precipitações pluviais subestimarem as medidas, sendo a diferença entre as previsões e as medidas diretamente proporcional ao aumento do período de antecedência da previsão. As previsões de temperatura do ar no Paraná tenderam a aumentar as variações entre os dias e, por outro lado, tenderam a reduzir a amplitude das variações durante os dias. No entanto, a redução das amplitudes térmicas durante o dia apresentou erros sistemáticos, pois tanto as temperaturas máximas do ar como as mínimas previstas foram maiores que as medidas.

No caso das diferenças sazonais, observouse que as subestimativas das previsões de precipitação pluvial se concentraram nos meses de dezembro a março, ou seja, durante o período recomendado para o cultivo de milho na região. Por outro lado, as superestimativas das precipitações pluviais com relação às medidas ocorreram nos meses de junho a setembro, implicando que essas superestimativas não devem ser problemáticas na aplicação de modelos para a previsão de rendimento de milho, visto que ocorrem em período distinto ao recomendado para o cultivo de milho na região. A sazonalidade das diferenças entre as temperaturas do ar previstas e as medidas também não deve ser problemática, pois as maiores diferenças de temperatura do ar também se concentraram nos meses de junho a setembro.

A partir da exposição sobre as previsões de precipitação pluvial e temperatura do ar geradas pelo Modelo Eta no Paraná, é possível inferir que a aplicação das previsões do modelo na previsão do rendimento de grãos de milho por modelos de cultura sofrerá limitações sem a implementação de rotinas que determinem e mitiguem o erro sistemático. As previsões meteorológicas geradas pelo Modelo Eta para Campo Grande, em Mato Grosso do Sul, apresentaram comportamento semelhante às previsões do Paraná, ou seja, redução da amplitude das precipitações pluviais previstas entre os dias e subestimativa das previsões de precipitação pluvial com relação às medidas. Portanto, é possível inferir que o Modelo Eta não preveja adequadamente as precipitações pluviais extremas para esse local. Quanto às previsões de temperatura do ar em Campo Grande, também foram mantidas as tendências de aumentar as amplitudes entre os dias e de reduzir a amplitude térmica diurna, mas com temperaturas máximas do ar previstas maiores que as medidas, semelhante ao Paraná. A sazonalidade das diferenças entre as previsões e as respectivas medidas de precipitação pluvial em Campo Grande também são semelhantes às verificadas para o Paraná. As previsões de precipitação pluvial geradas pelo Modelo Eta para os locais examinados em Minas Gerais tendem a ser distribuídas de forma mais regular temporalmente que as respectivas precipitações pluviais medidas. As precipitações pluviais previstas tendem a ser maiores que as respectivas medidas nos locais mais ao Sul e com climas de características mais temperadas, enquanto nos demais locais os resultados não permitem definir uma tendência geral, à exceção de Janaúba, em que as previsões tendem a subestimar a precipitação pluvial. Quanto à temperatura do ar, as previsões meteorológicas geradas pelo Modelo Eta tendem a aumentar as variações entre os dias, minimizando, contudo, as amplitudes térmicas diárias em razão dos maiores valores previstos para as temperaturas mínimas do ar.

Considerando o exposto sobre as previsões de precipitação pluvial e de temperatura do ar geradas pelo Modelo Eta para Minas Gerais, é possível inferir que o uso dessas previsões para a previsão do rendimento de grãos de milho resulte em superestimativas nas localidades mais ao Sul do Estado, em razão de as precipitações pluviais previstas serem maiores que as medidas. Nas demais localidades, os rendimentos de grãos estimados não devem ser afetados pelas previsões de precipitação pluvial e de temperatura do ar geradas pelo Modelo Eta, exceto em Janaúba, onde as precipitações pluviais previstas são menores que as precipitações pluviais medidas, acarretando em subestimativa do rendimento de grãos. Assim como em São Paulo, as previsões geradas pelo Modelo Eta são bastante influenciadas pelo local, dificultando a extrapolação espacial de suas previsões.

De modo geral, as previsões de precipitação pluvial geradas pelo Modelo Eta para os locais examinados em Goiás tendem a ser mais regularmente distribuídas no tempo e menores que as precipitações pluviais medidas, notadamente em Porangatu. A 
exceção foi para as previsões de precipitação pluvial em Itumbiara, porém, as diferenças entre as precipitações pluviais previstas e as respectivas precipitações pluviais medidas são inferiores a $4 \%$. Quanto à temperatura do ar, as previsões geradas pelo Modelo Eta tendem a aumentar as variações entre os dias, minimizando, contudo, as amplitudes térmicas diárias em razão dos maiores valores previstos para as temperaturas mínimas do ar. Essas observações gerais para Goiás também são dependentes do local. Entretanto, diferentemente de São Paulo e Minas Gerais, apresentam comportamentos semelhantes, o que possibilita a extrapolação espacial das previsões, à exceção de Itumbiara.

As previsões de precipitação pluvial, para o Estado da Bahia, geradas pelo Modelo Eta para Barreiras, são distribuídas mais regularmente no tempo que as respectivas precipitações pluviais medidas. No entanto, as precipitações pluviais previstas são menores que as respectivas precipitações pluviais medidas. Quanto à temperatura do ar, as previsões geradas pelo Modelo Eta tendem a reduzir as variações entre os dias e durante os dias em razão dos menores valores previstos para as temperaturas máximas e maiores valores previstos para as temperaturas mínimas. Assim, é possível inferir que as previsões do Modelo Eta em Barreiras são as piores dos 24 locais estudados e que sua imprecisão é diretamente proporcional ao período de antecedência da previsão. Observa-se também que o comportamento e as tendências das previsões geradas pelo Modelo Eta para Barreiras diferem do comportamento e das tendências verificados nos demais locais. Desse modo, qualquer extrapolação ou inferência das previsões realizadas nos demais locais para Barreiras fica prejudicada. Assim, conclui-se que não é possível aplicar os parâmetros climáticos gerados pelo Modelo Eta, sobretudo a precipitação pluvial, para estimativa do rendimento de grãos de milho por modelos de cultura. Quanto aos resultados similares, percebese que o erro aumenta do Sul para o Norte, ou seja, a aplicação em Santa Catarina e no Rio Grande do Sul deve ser ainda pior que no Paraná. Em contrapartida, na região Norte, deve-se ter cuidado em realizar as inferências porque a probabilidade de erro é muito grande.

\section{CONCLUSÕES}

Os resultados indicam que os dados gerados pelo Modelo ETA podem ser utilizados em previsões. No entanto, foram verificados erros sistemáticos nas previsões de temperaturas que podem ser removidos para que os valores corrigidos possam ser introduzidos nos modelos de culturas.

\section{FONTES DE AQUISIÇÃO}

a- As coordenadas verticais do modelo são definidas em MESINGER (1984), em que são consideradas uma pressão de referência adequadamente definida e a função da altura, sendo a pressão ao nível médio do mar igual a 1013,25hPa, a pressão à superfície, a pressão no topo do modelo e a altura da topografia. As versões de $40 \mathrm{~km}$ e $80 \mathrm{~km}$ possuem 38 camadas, sendo a resolução maior nos baixos níveis, que diminui com a altura; a primeira camada tem $20 \mathrm{~m}$ de espessura. Um máximo secundário ocorre próximo à tropopausa para melhor descrever as frentes em altos níveis.

b- Não foi objetivo comparar os valores de radiação estimados pelo Modelo Eta e os valores medidos.

\section{REFERÊNCIAS}

CHOU, S.C.; SILVA, J.M.G.A. Objective evaluation of Eta precipitation forecasts over South América. Climanálise, Cachoeira Paulista, SP, v.14, n.1, p.1-17, 1999.

CHOU, S.C. Modelo regional Eta. Climanálise. v.1, n.Ed. Especial, p.203-207, 1996.

CHOU, S.C. et al. Eta Model forecasts for the Venezuela flood event of December 1999. Revista Brasileira de Meteorologia, v.19, n.1, p.99-112, 2004.

CHOU, S.C. et al. Extended range runs using the Eta model over South America. Journal of Geophysical Research, v.105, n.5, p.10147-10160, 2000

CHOU, S.C. et al. Refinamento estatístico das previsões horárias de temperatura a $2 \mathrm{~m}$ do modelo Eta em estações do Nordeste do Brasil. Revista Brasileira de Meteorologia, v.22, n.3, 287-296, 2007.

CHOU,S.C. et al. Evaluation of seasonal precipitation forecasts over South America using Eta model. Nonlinear Processes in Geophysics, v.12, n.4, p.537-555, 2005.

DERECZYNSKI, C.P. et al. Avaliação do desempenho do modelo regional Eta utilizando um topografia de 30 segundos. In: CONGRESSO BRASILEIRO DE METEOROLOGIA, 11., 2000, Rio de Janeiro. Anais... Rio de Janeiro: Sociedade Brasileira de Meteorologia, 2000. 1 CD-ROM.

DUARTE, A.P; PATERNIANI, M.E.A.G.Z. Fatores bióticos e abióticos em cultivares de milho e estratificação ambiental: avaliação IAC/CATI/Empresas - 1999/2000. Campinas: Instituto Agronômico de Campinas, 2000. 150p.

MESINGER, F. et al. Eta model precipitation forecast for a period including tropical storm Alison. Weather and Forecasting, v.5, n.3, p.483-493, 1990.

SAULO, A.C. et al. Model characterization of the south american low-level folw during the 1997-1998 spring-summer season. Climate Dynamics, v.18, p.867-881, 2000.

SELUCHI, M.E.; CHOU, S.C. Intercambios de masas de aire entre latitudes tropicales y extratropicales de Sudamerica. Climanálise, v.14, n.1, p.46-70, 2003.

SELUCHI, M. et al. Analysis of three situations of the foehn effects over the Andes (zonda wind) using the Eta-CPTEC regional model. Weather and forecasting, v.18, 481-501p., 2003. 